\title{
Respuesta a la prueba de caminata de seis minutos en niños con riesgo cardiovascular
}

\author{
Response to the six-minute walk test in children with cardiovascular risk
}

\author{
Daniel Ciudad ${ }^{\mathrm{a}, \mathrm{c}, \mathrm{d}}$, Ester Arellano $^{\mathrm{c}}$, Patricia Díaz ${ }^{\mathrm{a}, \mathrm{d}}$, Rubén Donoso $^{\mathrm{a}}$, Yocelin Rivala \\ Valentina Rojas ${ }^{\mathrm{a}}$, Nicolás Sánchez ${ }^{\mathrm{a}}$, Marcelo Cano-Cappellacci ${ }^{\mathrm{a}} \mathrm{b}, \mathrm{d}$
}

\author{
aEscuela de Kinesiología, Universidad de Valparaíso, Chile \\ bDepartamento de Kinesiología, Universidad de Chile. Chile \\ 'Hospital Carlos Van Buren, Valparaíso, Chile \\ dKinesiólogo
}

Recibido: 20 de enero de 2020; Aceptado: 1 de junio de 2020

\section{¿Qué se sabe del tema que trata este estudio?}

La obesidad infantil es un problema de salud pública que demanda herramientas de valoración para avanzar en la prevención y pesquisa temprana de las enfermedades cardiovasculares en niños obesos. La prueba de caminata de seis minutos busca este objetivo.

\section{¿Qué aporta este estudio a lo ya conocido?}

La literatura valida la prueba de caminata de seis minutos como un instrumento para determinar el rendimiento aeróbico en niños obesos y definir su deterioro funcional cardiorrespiratorio. El estudio pone en duda esta aseveración y propone alternativas.

\section{Resumen}

El riesgo cardiovascular (RCV) se define como la posibilidad que tiene un sujeto de sufrir una enfermedad cardiovascular dentro de un determinado plazo de tiempo. Si bien la patología se hace sintomatica en etapa adulta, los cambios fisiopatológicos comienzan a desarrollarse en edades tempranas. Objetivo: Establecer la relación entre capacidad cardiorrespiratoria, estimada a través de la prueba de caminata de seis minutos (PC6M), y el RCV en niños con síndrome metabólico. Pacientes y Método: Se analizaron 42 niños, edad 5 a 15 años, que asistieron a la Unidad de Cardiología Infantil del Hospital Carlos Van Buren entre los años 2015 y 2017. Cada participante se categorizó de acuerdo al puntaje de RCV de Alustiza, que define 3 niveles de riesgo: bajo ( 0 a 6 puntos), medio ( 7 a 8 puntos) y alto ( 9 o más puntos), lo que se traduciría en una mayor probabilidad de desarrollar una enfermedad cardiovascular, y realizó concomitantemente la PC6M. Resultados: La edad media del grupo de niños fue de 10,9 $\pm 2,7$ años, con un Índice de Masa Corporal (IMC) $=31,0 \pm 4,6 \mathrm{~kg} / \mathrm{m}^{2}$ (z-score 3,2 \pm $0,7)$. Los niños recorren $75,2 \pm 8,9$ por ciento de la distancia teórica, con un porcentaje de frecuencia cardíaca de reserva $(\mathrm{FCR})=31,0 \pm 9,4$. No se observaron relaciones estadísticamente significativas entre RCV y PC6M. Conclusiones: No se encuentra relación entre la capacidad cardiorrespiratoria y RCV. Se cuestiona la utilidad de la PC6M para valorar el RCV en la población de estudio.
Palabras clave: Riesgo Cardiovascular; Puntaje de Alustiza; Prueba de Caminata de Seis Minutos;

Niños;

Síndrome Metabólico

Correspondencia:

Daniel Ciudad

daniel.ciudad@uv.cl 


\begin{abstract}
Introduction: Cardiovascular risk (CVR) is defined as the possibility of a subject suffering from cardiovascular disease within a certain period. Although the pathology appears in adult life, the physiopathological changes start to develop at an early age. Objective: To establish the relationship between cardiorespiratory capacity (CRC) and CVR in children with metabolic syndrome. Patients and Methods: We analyzed data corresponding to 42 children aged from 5 to 15 years who were seen at the Children's Cardiology Unit of the Carlos Van Buren Hospital between 2015 and 2017. Each participant was categorized according to the Alustiza's CVR score, which defines 3 levels of risk: low (0 to 6 points), medium ( 7 to 8 points) and high ( 9 or more points), which representing a greater probability of developing cardiovascular disease, and performed 6MWT. Results: The mean age of the children was $10.9 \pm 2.7$ years, body mass index $(\mathrm{BMI})=31.0 \pm 4.6 \mathrm{~kg} / \mathrm{m}^{2}(\mathrm{z}$-score $3.2 \pm 0.7)$, percentage of theoretical distance walked $=75.2 \pm 8.9$, and percentage of heart rate reserve $($ HRR $)=31.0 \pm 9.4$. There was no statistical association between 6MWT and CVR. Conclusions: There is no relationship between the cardiorespiratory capacity and the CVR. The use of the 6MWT is questioned as an instrument to assess CVR in the population under study.
\end{abstract}

Keywords:

Cardiovascular Risk;

Alustiza Score;

Six-Minute Walk Test;

Children;

Metabolic Syndrome

\section{Introducción}

Según la última Encuesta Nacional de Salud, en Chile 11 millones de personas (de 15 años y más) presentan algún factor de RCV: hipertensión 27,6 \%, obesidad $34,4 \%$, diabetes $12,3 \%$, tabaquismo $33,4 \%$, o sedentarismo $87 \%{ }^{1}$. El RCV se define como la posibilidad que tiene un sujeto de sufrir una enfermedad cardiovascular dentro de un determinado plazo de tiem$\mathrm{po}^{2}$. Esto va a depender de la cantidad de factores de riesgo que presente el individuo, los cuales pueden ser modificables o no. Dentro de los factores modificables destacan la obesidad, hipertensión arterial, consumo perdurable de una dieta aterogénica y hábitos, como la inactividad física, tabaquismo y alcoholismo, entre otras. Por otro lado, los factores genéticos son el gran pilar de los factores no modificables ${ }^{3}$. En la población infantil, actualmente ya existe constancia de estos factores que van a promover y establecer el RCV ${ }^{4,5}$, por lo tanto, es necesario pesquisar tempranamente el riesgo en esta población. De esta manera se logrará que ese individuo, cuando sea adulto, tenga una menor probabilidad de desarrollar alguna enfermedad cardiovascular.

A nivel mundial, 360 millones de niños y adolescentes, entre 5 y 19 años, presentan sobrepeso u obesidad, así como también, 41 millones de niños menores a 5 años $^{6}$. En Chile, existe una prevalencia de un $50,9 \%$ de sobrepeso y obesidad en niños de primer año básico ${ }^{7}$. Por otro lado, estudiando el rendimiento cardiovascular y potencia aeróbica máxima en niños de octavo básico, se observó que el $72 \%$ de ellos tienen un nivel insatisfactorio ${ }^{8}$. Además, se sabe que aquellos niños con sobrepeso u obesidad son los alumnos con una peor condición física en población escolar?.

Las pruebas cardiorrespiratorias tienen como objetivo evaluar la capacidad de captar, entregar y utili- zar el oxígeno por parte de los distintos componentes del cuerpo humano, durante el ejercicio y la actividad física. El indicador más confiable para la medición de la capacidad cardiorrespiratoria (CCR) es la cuantificación del consumo máximo de oxígeno $\left(\mathrm{VO}_{2 \text { máx }}\right)$. Existen dos modalidades para la medición de este indicador, una es a través del análisis directo de gases en laboratorio, y la otra es a través de pruebas indirectas de campo que estiman el $\mathrm{VO}_{2 \text { máx }}$. Dentro de estas últimas se encuentran las pruebas de ejercicio cardiorrespiratorio. La más utilizada y recomendada en niños y adolescentes es la prueba de lanzadera de $20 \mathrm{~m}$ y, si la población es de riesgo, la PC6M, dado que es una prueba sencilla, segura, confiable y de gran valide $z^{10-13}$.

El objetivo del estudio fue determinar si existe relación entre la capacidad cardiorrespiratoria, estimada a través de la PC6M y el RCV en niños con síndrome metabólico, con el fin de validar la prueba como herramienta de apoyo en la prevención y pesquisa de las enfermedades cardiovasculares en este grupo etario.

\section{Pacientes y Método}

Estudio descriptivo retrospectivo correlacional no probabilístico, correspondiente a una base de datos anónima de 71 niños de ambos sexos, entre 5 y 15 años, con antecedentes de hipotiroidismo, hipercolesterolemia, dislipidemia, resistencia a la insulina, obesidad y sobrepeso. Todos pertenecientes al programa de RCV del policlínico de cardiología infantil del Hospital Carlos Van Buren (HCVB) de Valparaíso entre los años 2015 y 2017. Se excluyeron en el análisis los datos de aquellos niños con patologías cardíacas asociadas, uso de fármacos cardiovasculares, presencia de enfermedades musculo esqueléticas o limitantes para 
la marcha, asma bronquial no controlada, antecedente de hospitalización o consulta a servicio de urgencia las últimas cuatro semanas (figura 1). Cuarenta y dos registros fueron seleccionados, donde se analizó: edad, sexo, peso, talla e IMC z-score. Cada participante fue categorizado de acuerdo al puntaje de RCV de Alustiza (tabla 1), que define 3 niveles de riesgo: bajo ( 0 a 6 puntos), medio ( 7 a 8 puntos) y alto ( 9 o más puntos), lo que se traduciría en una mayor probabilidad de desarrollar una enfermedad cardiovascular ${ }^{14}$. Se realizó la PC6M según protocolo de la American Thoracic Society (ATS) para estimar su capacidad cardiorrespiratoria $^{11-13}$. El objetivo de dicha prueba es cubrir la mayor distancia posible en seis minutos. Se debe caminar lo más rápido posible sin correr, un tramo de 30 metros de largo, el cual debe ser recorrido en forma cíclica tantas veces pueda el sujeto durante el tiempo señalado. Previo a la prueba se evaluó a cada sujeto: peso y talla con una báscula con tallímetro SECA modelo 220. Con un monitor CONTEC modelo CMS5000, se evaluó su frecuencia cardíaca de reposo $\left(\mathrm{FC}_{\mathrm{R}}\right)$, saturación de oxí- geno $\left(\mathrm{SatO}_{2}\right)$ y presión arterial (PA). El sujeto estuvo 10 min sentado en reposo antes de las respectivas mediciones. Sensación subjetiva de fatiga de extremidades inferiores (SSF- EI) y sensación de disnea se valoraron a través de la escala de Borg modificada (rango 0 a 10$)^{15}$. Las mediciones se repitieron inmediatamente terminada la prueba, a los 5 y $10 \mathrm{~min}$ de reposo, registrando además la frecuencia cardíaca máxima alcanzada posterior al ejercicio $\left(\mathrm{FC}_{\text {Máx. Trabajo }}\right)$. Se registró la frecuencia cardíaca máxima teórica $\left(\mathrm{FC}_{\text {Máx. Teórica }}\right)$ y el porcentaje de la frecuencia cardíaca de reserva (FCR) según las siguientes fórmulas:

$\mathrm{FC}_{\text {Máx. Teórica }}=210-\left(\operatorname{Edad}^{*} 0,65\right) l p m$ y\%FCR $\frac{\mathrm{FC}_{\text {Máx. Trabajo }}-\mathrm{FC}_{\text {Reposo }}}{\mathrm{FC}_{\text {Máx. Teórica }}-\mathrm{FC}_{\text {Reposo }}} \mathrm{x} 100^{11,16}$,

a fin de monitorizar la respuesta cardíaca y niveles de intensidad del ejercicio físico. Para el cálculo de la distancia máxima teórica a recorrer en la PC6M se utilizó los valores de Gatica et al $^{11}$, y para la estimación del $\mathrm{VO}_{2 \text { máx. }}$ se aplicó la fórmula de Jalili et $\mathrm{al}^{12}$.

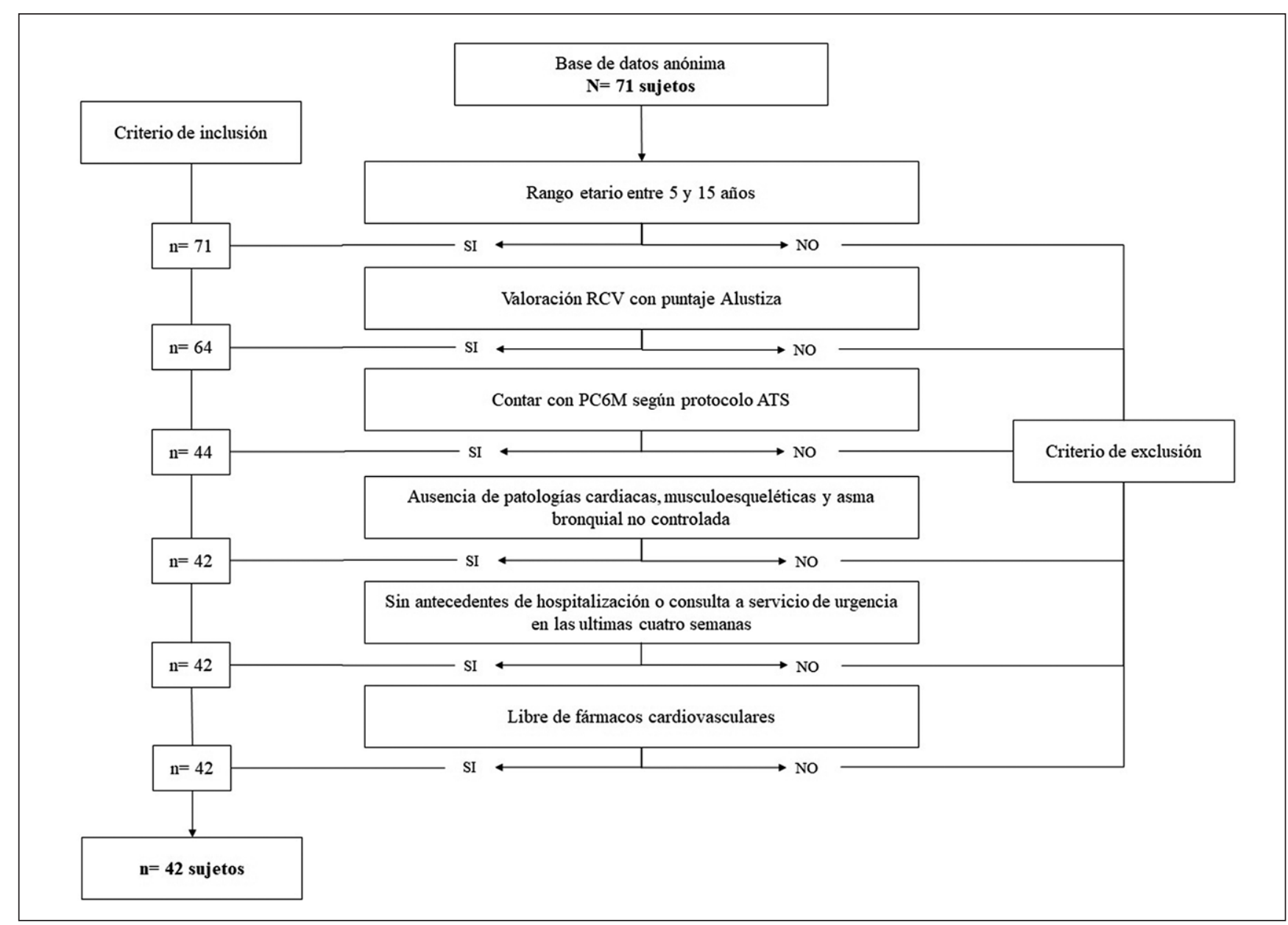

Figura 1. Selección de la muestra. RCV: riesgo cardiovascular. PC6M: prueba de caminata de seis minutos. 


\section{Análisis estadístico}

Para el análisis estadístico se utilizó el programa IBM SPSS Statistics software 24.0. La estadística descriptiva fue expresada en media con su respectiva desviación estándar y mediana [mínimo - máximo]. Se realiza la prueba de correlación de Spearman (rho) debido a la naturaleza de la variable de puntaje Alustiza, que no se distribuye de manera normal por ser una variable discreta.

Tabla 1. Puntaje de RCV de Alustiza et al

\begin{tabular}{|c|c|c|}
\hline Variable & Valor & Puntuación \\
\hline Edad & $\begin{array}{l}2 \text { a } 5 \text { años } \\
6 \text { a } 12 \text { años } \\
>13 \text { años }\end{array}$ & $\begin{array}{l}0 \text { puntos } \\
2 \text { puntos } \\
3 \text { puntos }\end{array}$ \\
\hline Sexo & $\begin{array}{l}\text { Mujer } \\
\text { Varón }\end{array}$ & $\begin{array}{l}0 \text { puntos } \\
2 \text { puntos }\end{array}$ \\
\hline Antecedentes familiares & $\begin{array}{l}\text { Ausentes } \\
\text { (+) Bioquímicos } \\
\text { (+) Clínicos }\end{array}$ & $\begin{array}{l}0 \text { puntos } \\
2 \text { puntos } \\
4 \text { puntos }\end{array}$ \\
\hline Ejercicio & $\begin{array}{l}>2 \mathrm{~h} / \text { día y } T V<3 \mathrm{~h} / \text { día } \\
<2 \mathrm{~h} / \text { día y } T V>3 \mathrm{~h} / \text { día }\end{array}$ & $\begin{array}{l}0 \text { puntos } \\
1 \text { punto }\end{array}$ \\
\hline Tabaco/Alcohol & $\begin{array}{l}\text { No } \\
\text { Sí }\end{array}$ & $\begin{array}{l}0 \text { puntos } \\
1 \text { punto }\end{array}$ \\
\hline Obesidad (IMC) & $\begin{array}{l}<\text { Percentil } 95 \\
>\text { Percentil } 95\end{array}$ & $\begin{array}{l}0 \text { puntos } \\
1 \text { punto }\end{array}$ \\
\hline Presión arterial & $\begin{array}{l}<\text { Percentil } 95 \\
>\text { Percentil } 95\end{array}$ & $\begin{array}{l}0 \text { puntos } \\
1 \text { punto }\end{array}$ \\
\hline Colesterol & $\begin{array}{l}\text { CT: 150-199, LDL 100-109 } \\
\text { CT: 200-220, LDL 110-130 } \\
\text { CT: 221-230, LDL 131-160 } \\
\text { CT: 231-280, LDL 161-190 } \\
\text { CT > 281, LDL > } 190\end{array}$ & $\begin{array}{l}0 \text { punto } \\
1 \text { punto } \\
2 \text { puntos } \\
3 \text { puntos } \\
6 \text { puntos }\end{array}$ \\
\hline
\end{tabular}

Puntuación: Total puntos 19; riesgo bajo: 0-6 ; riesgo medio: 7-8; riesgo alto: 9 o más. RCV: riesgo cardiovascular. IMC: indice de masa corporal. CT: colesterol total.

Tabla 2. Caracterización de los sujetos en estudio

\begin{tabular}{lccc}
\hline & $\begin{array}{c}\text { Total } \\
(\mathrm{n}=42)\end{array}$ & $\begin{array}{c}\text { Hombres } \\
(\mathrm{n}=18)\end{array}$ & $\begin{array}{c}\text { Mujeres } \\
(\mathrm{n}=24)\end{array}$ \\
\hline Edad (años) & $10,90 \pm 2,72$ & $10,61 \pm 3,16$ & $11,13 \pm 2,38$ \\
Peso $(\mathrm{kg})$ & $70,49 \pm 18,31$ & $70,61 \pm 20,18$ & $70,40 \pm 17,22$ \\
Talla $(\mathrm{m})$ & $1,49 \pm 0,12$ & $1,50 \pm 0,15$ & $1,49 \pm 0,09$ \\
IMC (kg/m²) & $31,04 \pm 4,58$ & $30,58 \pm 4,53$ & $31,40 \pm 4,68$ \\
IMC $z$-score & $3,20 \pm 0,66$ & $3,43 \pm 0,78$ & $3,08 \pm 0,51$ \\
\hline
\end{tabular}

IMC: índice de masa corporal.

\section{Resultados}

La muestra correspondió a 18 sujetos de sexo masculino y 24 de sexo femenino, la caracterización de los sujetos en estudio se muestra en la tabla 2. Los resultados obtenidos en la PC6M y puntaje Alustiza se muestran en la tabla 3. En relación a la categorización de su RCV, sólo 3 sujetos se encuentran dentro de la categoría de riesgo medio (7-8 pts.) y los 39 restantes en riesgo alto ( 9 o más pts.). Todos los sujetos completaron la PC6M sin mayores inconvenientes en cuanto a su comportamiento cardiorrespiratorio y sólo 2 sujetos registran una detención durante el desarrollo

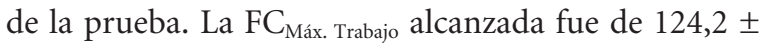
$11,1 \mathrm{lpm}$, para una distancia total recorrida (DR) de $433,4 \pm 58,6 \mathrm{~m}$. Por otro lado, el porcentaje de FCR se sitúa en 31,0 \pm 4,6 al momento de finalizar la prueba. El porcentaje de saturación de oxígeno fue de 97,2 \pm 1,5, presión arterial sistólica (PAS) fue de 125,2 $\pm 13,4$ $\mathrm{mmHg}$ y presión arterial diastólica (PAD) fue de 68,1 $\pm 10,4 \mathrm{mmHg}$. El $\mathrm{VO}_{2 \text { máx. }}$ estimado fue de 15,8 $\pm 4,7$ $\mathrm{ml} / \mathrm{kg} / \mathrm{min}$. La mediana de SSF-EI fue de 0 [0-5] y de disnea fue de 0 [0-2].

Analizando el comportamiento de las variables en estudio (tabla 4), se observa valores de correlación entre DR y: Edad $=0,44 ;$ Talla $=0,50 ; \mathrm{FC}_{\text {Máx teórica }}=0,43$; Distancia recorrida teórica $(\%)=0,53 ; \mathrm{PAD}=0,43$; $\mathrm{VO}_{2 \text { máx. }}=0,67$.

El grado de correlación entre las variables DR y RCV es inexistente, con un valor Rho de 0,004; p de 0,98 .

\section{Discusión}

Los resultados del estudio muestran que no hay una relación entre la DR por los niños en la PC6M y el RCV. Sin embargo, los niños de la muestra recorren menos distancia en comparación a la población pediátrica chilena sana ${ }^{11}$ y presentan un alto RCV. Si bien el rendimiento de la prueba puede verse afectado por otros factores como la talla, género, estado de salud entre otros ${ }^{13}$, nos preguntamos sobre la utilidad de la PC6M para evaluar la CCR en la población de estudio. La PC6M ha demostrado ser una excelente herramienta para medir la CCR en poblaciones pediátricas con enfermedades crónicas ${ }^{11}$, sin embargo en relación a la obesidad, existe poca evidencia científica en nuestro país. Se cuestiona la aplicación clínica de las pruebas de marcha para la valoración de la CCR en niños sin manifestaciones clínicas. La razón radica en que las pruebas de caminata son pruebas de esfuerzo submáximo. Durante el ejercicio submáximo, la capacidad de transporte y utilización del oxígeno son cubiertos perfectamente por el organismo durante un tiempo 
relativamente prolongado, por lo que la CCR no es explorada en su totalidad. Por el contrario, una prueba de ejercicio progresivo máximo implica un aumento considerable de la demanda de oxígeno, poniendo a prueba todas aquellas funciones fisiológicas que determinan una rápida respuesta del sistema de transporte de oxígeno, permitiendo evaluar de forma más precisa la $\mathrm{CCR}^{10,17}$.

Se considera que la condición física relacionada con la salud, está asociada a la CCR, entendiendo ésta como: "una medida de la capacidad de realizar actividad física y/o ejercicio físico integrando la mayoría de las funciones involucradas en el movimiento corpo$\mathrm{ral}^{\prime 17}$. Entre más exigente sea la prueba, más certera es la evaluación del comportamiento de esta variable en la condición de salud de los niños con $\mathrm{RCV}^{10}$. En base a esta premisa, se estudió el comportamiento de la CCR en otra prueba de caminata, la prueba de caminata de carga progresiva PCCP (Incremental Shuttle Walking Test), donde efectivamente los niños tienen mejor rendimiento que en la PC6M, con un porcentaje de FCR mayor al 90\%. Para lograr esta condición los autores recomiendan, en la población pediátrica, agregar tres niveles adicionales de velocidad a la prueba original y permitir que el niño corra, en busca de un esfuerzo progresivo máximo ${ }^{15,18,19}$.

Otra posible explicación a la baja correlación encontrada entre PC6M y el RCV medido a través de
Tabla 4. Correlación entre distancia total recorrida y parámetros cardiorrespiratorios

\begin{tabular}{|c|c|c|}
\hline & Correlación & p-valor \\
\hline Edad (años) & 0,44 & $* 0,005$ \\
\hline Peso (kg) & 0,36 & $* 0,019$ \\
\hline Talla (m) & 0,50 & $* 0,001$ \\
\hline IMC $\left(\mathrm{kg} / \mathrm{m}^{2}\right)$ & 0,13 & 0,418 \\
\hline IMC z-score & 0,40 & $* 0,009$ \\
\hline Distancia teórica (\%) & 0,53 & ${ }^{*}<0,001$ \\
\hline $\mathrm{FC}_{\text {Máx. de trabajo }}$ alcanzado (Ipm) & 0,11 & 0,504 \\
\hline $\mathrm{FC}_{\text {Máx. teórica }}(\mathrm{lpm})$ & 0,43 & ${ }^{*} 0,004$ \\
\hline $80 \%$ de la $\mathrm{FC}_{\text {Máx. de trabajo }}(\mathrm{lpm})$ & 0,40 & ${ }^{*} 0,008$ \\
\hline FC reserva (\%) & 0,35 & $* 0,022$ \\
\hline Sat $\mathrm{O}_{2}(\%)$ & 0,21 & 0,434 \\
\hline PAS (mmHg) & 0,35 & ${ }^{*} 0,022$ \\
\hline PAD (mmHg) & 0,43 & $* 0,004$ \\
\hline $\mathrm{VO}_{2 \text { máx. }}(\mathrm{mL} / \mathrm{kg} / \mathrm{min})$ & 0,67 & ${ }^{*}<0,001$ \\
\hline Puntaje Alustiza (ptje.) & (rho) 0,004 & 0,982 \\
\hline Disnea (Borg) & (rho) 0,15 & 0,313 \\
\hline SSF- El (Borg) & (rho) 0,21 & 0,182 \\
\hline
\end{tabular}

p-valor= referido al valor de significancia de la prueba. IMC: índice de masa corporal; FC: Frecuencia cardiaca; $\mathrm{SatO}_{2}$ : Saturación de oxígeno; PAS: Presión arterial sistólica; PAD: Presión arterial diastólica; $\mathrm{VO}_{2}$ máx: Consumo máximo de oxígeno; SSF-EI: sensación subjetiva de fatiga extremidades inferiores. *Se considera un valor $p<0,05$.

Tabla 3. Media y desviación de resultados de prueba de caminata de 6 minutos y puntaje de riesgo cardiovascular

\begin{tabular}{|c|c|c|c|c|}
\hline & Total $(n=42)$ & Hombres $(n=18)$ & Mujeres $(n=24)$ & $\mathrm{p}$-valor \\
\hline $\mathrm{DR}(\mathrm{m})$ & $433,4 \pm 58,6$ & $430,5 \pm 47,1$ & $435,5 \pm 66,9$ & 0,27 \\
\hline Distancia teórica (m) & $575,9 \pm 22,9$ & $565,89 \pm 26,6$ & $583,32 \pm 16,6$ & $\star 0,02$ \\
\hline Distancia teórica (\%) & $75,2 \pm 9$ & $76,0 \pm 6,7$ & $74,5 \pm 10,4$ & 0,52 \\
\hline FC Máx. de trabajo alcanzado (Ipm) & $124,17 \pm 11,11$ & $122,67 \pm 11,57$ & $125,29 \pm 10,86$ & 0,75 \\
\hline $\mathrm{FC}$ Máx. teórica $(\mathrm{lpm})$ & $203,34 \pm 3,18$ & $203,08 \pm 2.06$ & $203,54 \pm 3,85$ & 0,62 \\
\hline $80 \%$ de la FC Máx. de trabajo (Ipm) & $162,23 \pm 1,45$ & $162,43 \pm 1,64$ & $162,08 \pm 1,30$ & 0,45 \\
\hline FC reserva (\%) & $31,03 \pm 9,42$ & $29,88 \pm 9,78$ & $31,90 \pm 9,25$ & 0,68 \\
\hline Sat $\mathrm{O}_{2}(\%)$ & $97,24 \pm 1,54$ & $97,44 \pm 1,25$ & $97,08 \pm 1,74$ & 0,74 \\
\hline PAS (mmHg) & $125,24 \pm 13,38$ & $126,11 \pm 13,95$ & $124,58 \pm 13,20$ & 0,36 \\
\hline PAD (mmHg) & $68,14 \pm 10,37$ & $67,94 \pm 9,08$ & $68,29 \pm 11,43$ & 0,1 \\
\hline \multirow[t]{2}{*}{$\mathrm{VO}_{2 \text { máx. }}(\mathrm{mL} / \mathrm{kg} / \mathrm{min})$} & $15,80 \pm 4,71$ & $15,73 \pm 4,29$ & $15,85 \pm 5,09$ & 0,07 \\
\hline & & \multicolumn{3}{|c|}{ Mediana (Mínimo-Máximo) } \\
\hline Puntaje Alustiza (ptje.) & & $11(7-14)$ & & \\
\hline Disnea (Borg) & & $0(0-2)$ & & \\
\hline SSF- El (Borg) & & $0(0-5)$ & & \\
\hline
\end{tabular}

p-valor= referido a comparación entre valores de hombres y mujeres como diferencias significativas, según prueba t-Student. DR: Distancia recorrida; FC: Frecuencia cardiaca; $\mathrm{SatO}_{2}$ : Saturación de oxígeno; PAS: Presión arterial sistólica; PAD: Presión arterial diastólica; $\mathrm{VO}_{2}$ máx: Con- $^{2}$ sumo máximo de oxígeno; SSF-El: sensación subjetiva de fatiga extremidades inferiores. *Se considera un valor $p<0,05$. 
la Escala de Alustiza, apunta directamente al mismo instrumento como medio de tamizaje de niños con riesgo metabólico. Si bien los autores declaran la falta de potencia estadística en sus resultados, nuestras observaciones van dirigidas principalmente a la metodología de evaluación y puntaje asignado para la variable "ejercicio". Alustiza y cols. consultan por el nivel de actividad física del niño en base a las horas/ día que éste dedica o permanece frente a un televisor, categorizando su condición de sedentario o no. Esto no necesariamente representa la condición física del menor, relacionada con fuerza, resistencia muscular, flexibilidad, velocidad, agilidad y CCR que son aspectos que explora la PC6M, por lo cual sería atendible la poca relación con la PC6M. Por otro lado, dentro del puntaje total de la escala, al variable ejercicio solo contempla un máximo de un punto ${ }^{14}$. Nos permitimos citar el trabajo de Touluse y cols. quienes utilizando calorimetría indirecta, monitoreo de frecuencia cardíaca y espectroscopía de infrarrojo cercano, durante ejercicios incrementales, están estudiando pruebas de campo confiables para evaluar la condición física en niños obesos ${ }^{20}$.

López et $\mathrm{al}^{21}$ realizaron el primer esfuerzo en caracterizar la respuesta cardiorrespiratoria de niños obesos a través de la PC6M, concluyendo que efectivamente los niños obesos recorren menos distancia que un niño normopeso y que la CCR se observaba limitada frente a la prueba que exigió un esfuerzo físico de hasta un $77 \%$ de la FCR. Nuestro estudio obtiene un 31\% para este comportamiento, muy similar a lo encontrado por Gatica et $\mathrm{al}^{11}$, cuando realiza la PC6M en 192 niños sanos. Bajo esta mirada y frente al mismo criterio (\% FCR), los niños obesos de nuestra investigación presentan el mismo comportamiento que niños sanos, no así los niños del estudio de López. La explicación de lo observado podría estar en que su estudio realiza una PC6M que permite al evaluador acompañar al niño durante la caminata, lo que no es parte del protocolo aceptado internacionalmente donde se establece que el paciente debe ir solo ${ }^{13}$. Si revisamos la literatura internacional, la PC6M es recomendada para su uso en niños obesos y el rendimiento mostrado se encuentra alrededor del 85\% en comparación con niños sanos normo peso. Sin embargo al observar el comportamiento de la frecuencia cardiaca, la sensación subjetiva de esfuerzo y el consumo oxígeno de los niños de las investigaciones, volvemos a constatar que la prueba se aleja considerablemente de un esfuerzo físico moderado, al igual que en nuestro estudio ${ }^{22,23}$.

Al analizar el comportamiento de las variables fisiológicas frente a la PC6M, surge la interrogante ¿por qué los niños presentan un bajo rendimiento en esta prueba? La respuesta podría estar asociada a otros factores, por ejemplo, una falta de motivación y/o concentración del niño frente al desarrollo de la prueba. Vilchez et $\mathrm{al}^{24}$, investigaron el clima motivacional en las clases de educación física, en donde concluyeron que los escolares que presentan un mayor patrón de actividad físico-deportiva, perciben un mayor clima motivacional, es decir, existiría una relación directamente proporcional entre la motivación y el rendimiento físico. Desde lo práctico, el logro de metas dentro de la psicología infantil, determinaría en gran parte el comportamiento frente a una prue$\mathrm{ba}^{25}$, por ende, se podría decir que la PC6M, al mantener una velocidad constante de marcha durante seis minutos, es una prueba monótona para el niño, que carece de objetivos competitivos como se expresaría en un juego, lo que va a afectar directamente el desempeño final.

Como debilidad de nuestro estudio es importante destacar la baja cantidad de niños que lograron completar el estudio de RCV y realizar la PC6M, Por ello los resultados obtenidos deben ser interpretados en relación a la muestra en estudio, no siendo posible que sean extrapolados a la población general. Sin embargo consideramos que el aporte de nuestro artículo es volver a revisar el tema del RCV en niños obesos y cómo se está evaluando su deterioro funcional cardiorrespiratorio.

Finalmente y en base a lo expuesto, es difícil apreciar las consecuencias de los factores de RCV en edades tempranas, producto de que la mayoría de los eventos cardiovasculares se presentan en la adultez. He aquí la importancia de hallar una herramienta confiable que apoye la pesquisa temprana y prevención de las enfermedades cardiovasculares en niños obesos.

\section{Conclusión}

No se encontro correlación entre la capacidad cardiorrespiratoria estimada a través de la PC6M y el respectivo puntaje de RCV de Alustiza. El comportamiento de variables fisiológicas en los niños, antes y después de la prueba demuestran un bajo esfuerzo físico exigido para cubrir la prueba.

\section{Responsabilidades Éticas}

Protección de personas y animales: Los autores declaran que los procedimientos seguidos se conformaron a las normas éticas del comité de experimentación humana responsable y de acuerdo con la Asociación Médica Mundial y la Declaración de Helsinki.

Confidencialidad de los datos: Los autores declaran 
que han seguido los protocolos de su centro de trabajo sobre la publicación de datos de pacientes.

\section{Derecho a la Privacidad y Consentimiento Informa-}

do: Los autores declaran que la información ha sido obtenida de datos previos en forma anonimizada, por lo cual el Comité de Ética de Investigación en uso de sus facultades, ha eximido de la obtención de un consentimiento informado, lo cual consta en el acta respectiva.

\section{Conflicto de intereses}

Los autores declaran no tener conflicto de intereses.

\section{Agradecimientos}

Agradecemos al kinesiólogo Manuel Vásquez Estaig, quién voluntariamente contribuyó a esta investigación, dando el apoyo técnico profesional necesario para su éxito.

\section{Referencias}

1. Margozzini P, Passi A. Encuesta Nacional de Salud, ENS 2016-2017: un aporte a la planificación sanitaria y políticas públicas en Chile. ARS Médica 2018;43(1):30-34.

2. Alegría E, Alegría A, Alegría E. Estratificación del riesgo cardiovascular: importancia y aplicaciones. Rev Esp Cardiol Supl. 2012;12(C):8-11.

3. Norte A, Sansano M, Martínez M, Sospedra I, Hurtado J, Moncada R. Estudio de factores de riesgo de enfermedad cardiovascular en trabajadores universitarios españoles. Nutr Hosp. 2016;33(3):644-8.

4. Escudero G, Morales L, Valverde C, et al. Riesgo cardiovascular en población infantil de 6 a 15 años con obesidad exógena. Rev Med Inst Mex Seguro Soc 2014; 52 (Supl 1); S58-S63.

5. Sapunar J, Aguilar-Farías N, Navarro J, et al. Alta prevalencia de dislipidemias y riesgo aterogénico en una población infanto-juvenil. Rev Med Chile 2018;146;1112-22.

6. World Health Organization. Obesidad y Sobrepeso [Internet]. 2018 [Acceso 15 de Junio 2019]. Disponible en: https://www. who.int/news-room/fact-sheets/detail/ obesity-and-overweight.

7. Junta Nacional de Auxilio Escolar y Becas [Internet] 2017. [Acceso 5 de Noviembre 2019]. Disponible en: https://www.junaeb.cl/wp-content/ uploads/2013/03/Informe-MapaNutricional-2017-FINAL.pdf.

8. Agencia de calidad de la Educación. Informe de resultados Estudio Nacional Educación Física $8^{\circ}$ básico [Internet] 2015. [Acceso 15 de Junio 2019]. Disponible en: http://archivos.
agenciaeducacion.cl/Informe_Nacional_ EducacionFisica2015.pdf.

9. Muros J, Cofre-Bolados C, Zurita-Ortega F, Castro-Sánchez M, Linares-Manrique M, Chacón Cuberos R. Relación entre condición física, actividad física y diferentes parámetros antropométricos en escolares de Santiago (Chile). Nutr Hosp. 2016;33(2):314-8.

10. Lang J, Tomkinson G, Janssen I, et al. Making a case for cardiorespiratory fitness surveillance among children and youth. Exerc Sport Sci Rev 2018;46(2):66-75.

11. Gatica D, Puppo H, Villarroel G, et al. Valores de referencia del test de marcha de seis minutos en niños sanos. Rev Med Chile 2012;140:1014-21.

12. Jalili M, Nazem F, Sazvar A, Ranjbar K. Prediction of Maximal Oxygen Uptake by Six-Minute Walk Test and Body Mass Index in Healthy Boys. J Pediatr. 2018;200:155-9.

13. Zenteno D, Puppo H, González R, Kogan $R$. Test de marcha de 6 minutos en pediatría. Neumol Pediatr. 2007;109-14.

14. Arnaiz P, Pino F, Marín A, et al. Validación de un puntaje de riesgo cardiovascular en niños españoles aplicado a una población de escolares de Santiago de Chile. Rev Med Chile 2010;138:1226-31.

15. Torres R, Zenteno D, Rodríguez I, et al. Guías de rehabilitación respiratoria en niños con enfermedad respiratorias crónicas: actualización 2016. Neumol Pediatr. 2016;11(3):114-31.

16. Strath S, Swartz A, Basset D, O'Brien W, King G, Ainswortz B. Evaluation of heart rate as a method for assessing moderate intensity physical activity. Med Sci Sports Exerc. 2000;32:S465-S470.

17. Martínez-Vizcaino V, Sánchez-López M. Relación entre actividad física y condición física en niños y adolescentes. Rev Esp Cardiol. 2008;61(2):108-11.

18. Ciudad D, Díaz P, Soto C, Orellana J. Prueba de caminata de carga progresiva (Incremental Shuttle Walking Test) en niños sanos. Rev Chil Enferm Respir. 2018;34(3):160-4.

19. Lanza F, Zagatto E, Cunha J, et al. Reference Equation for the Incremental Shuttle Walk Test in Children and Adolescents. J Pediatr 2015;167(5):105761.

20. Touluse L, Baquet G, Heyman E, et al. Respiratory responses and rating of perceived exertion of severely obese adolescents during continuous and intermittent graded walking protocols: Application to cardiorespiratory field tests. J Sports Sci. 2020;1-9. DOI: 10.1080/02640414.2020.1738701.

21. López A, Sotomayor L, Álvarez M, et al. Rendimiento aeróbico en niños obesos de 6 a 10 años. Rev Chil Pediatr 2009;80(5):444-50.

22. Morinder G, Mattsson E, Sollander C, Marcus C, Larsson U. Six-minute walk test in obese children and adolescents: reproducibility and validity. Physiother. Res Int. 2009;14(2):91-104.

23. Valerio G, Gallarato V, D'Amico O, et al. Perceived Difficulty with Physical Tasks, Lifestyle, and Physical Performance in Obese Children. Bio Med Res Int. 2014;17.

24. Vílchez P, Ruiz F. Clima motivacional en Educación Física y actividad físicodeportiva en el tiempo libre en alumnado de España, Costa Rica y México. Retos 2015;29:195-200.

25. Gutiérrez M, López E. Motivación, comportamiento de los alumnos y rendimiento académico. J Study Educ Dev 2012;35(1):61-72. 